\title{
Rotorcraft Gearbox Foundation Design by a Network of Optimizations
}

\author{
Geng Zhang ${ }^{1}$ and Ricardo Sbragio ${ }^{2}$ \\ Michigan Engineering Services, LLC, Ann Arbor, MI, 48108 \\ Nickolas Vlahopoulos ${ }^{3}$ \\ University of Michigan, Ann Arbor, MI, 48108
}

\begin{abstract}
Rotorcraft vehicle design is a complex process requiring interactions and exchange of information among multiple disciplines such as fatigue, strength, propulsion, noise, safety, maintenance, manufacturing, etc. Simulation models are employed for assessing and potentially improving a vehicle's performance in individual technical areas. The vehicle's characteristics influence the performance in all different attributes. Challenges arise when designing a vehicle for improving mutually competing objectives, satisfying constraints from multiple engineering disciplines, and determining a single set of values for the vehicle's characteristics. It is of interest to engage simulation models from the various engineering disciplines in an organized and coordinated manner for determining a design configuration that provides the best possible performance in all disciplines. This paper presents an approach that conducts optimization analysis for a complex system by coordinating operations and exchange of data and information through a network of optimizations. The presented approach provides an organized and seamless environment that captures the implications of design changes from a particular discipline to all other disciplines. It is possible to share design variables among disciplines and thus identify the overall direction that design variables should follow based on objectives and constraints from multiple and often mutually competing requirements. The multi-level optimization algorithm that drives the complex system design is presented first. A rotorcraft example that demonstrates the operation of this integrated design environment is discussed. The mass of the gearbox support frame is minimized while at the same time the performance in structural acoustics and crashworthiness is optimized.
\end{abstract}

\section{Introduction}

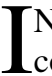
$\mathrm{N}$ order to be effective and maximize the weight and cost savings when designing a vehicle, the efforts must be concurrent considering multiple engineering disciplines in parallel (i.e. durability, crashworthiness, etc.). In this manner, it is possible to account for the effects of structural changes across disciplines and improve the performance while the structure is being configured. A flexible Multi-discipline Design Optimization (MDO) capability must be available for driving simultaneously multiple separate optimization analyses, facilitating the exchange of data among the disciplines, and accounting for the impact of changes introduced by a particular discipline to all others. The literature on MDO methods and applications is rich and representative references are [1-3]. The MDO term has been used for several different ways of considering multiple disciplines in an optimization process. In single objective optimizations it is used for indicating that the constraints are evaluated based on performance from different disciplines. In sequential optimizations (representative of a design spiral approach) it is used for reflecting that each optimization is associated with a different discipline. It is also used in multi-objective optimizations to indicate that multiple disciplines are considered when defining the cumulative single objective function which combines the performance metrics from the various disciplines. A new multi-level MDO optimization algorithm is presented in this paper for guiding in parallel a network of optimizations. It allows for solving simultaneously multiple individual optimizations for each discipline with separate objective functions and constraints, while at the

\footnotetext{
${ }^{1}$ Research and Development Engineer, 2890 Carpenter Road, Suite 1900, Ann Arbor, MI 48108.

${ }^{2}$ Research and Development Engineer, 2890 Carpenter Road, Suite 1900, Ann Arbor, MI 48108.

${ }^{3}$ Professor, NA\&ME Dept, 2600 draper Drive, Ann Arbor, MI 48109, AIAA Member.
} 
same time pursuing an overall system level weight or cost objective. A rotorcraft gearbox foundation design analysis is presented as an example of applying the new MDO algorithm in rotorcraft applications. The mass of the gearbox support frame is minimized while the performances under crash landing and structural acoustic considerations are optimized in parallel. The Hybrid Finite Element Analysis (hybrid FEA) [4-6] is employed for the structural acoustic computations. This method combines conventional finite elements for modeling the frame structure and the in-plane behavior of panels, with energy finite elements for modeling the flexible behavior of the panels. The input power in the rotorcraft structure is computed by the hybrid FEA for a given mechanical excitation; it comprises a metric of the structural vibration and of the interior noise generated from the gearbox excitation. The objective of the structural-acoustic optimization is to minimize the input power. The crashworthiness optimization minimizes the maximum dynamic stress encountered in the gearbox base when the helicopter is dropped to a rigid ground with an impact velocity of $30 \mathrm{~m} / \mathrm{s}$. This application is a challenging one because the system level objective of minimizing the gearbox mass is competing with the discipline level optimizations which require an increase in the mass of the gearbox base for reducing the input power and the maximum dynamic stress. Nevertheless, the new MDO algorithm is capable of identifying an optimum configuration that achieves improvements in all three performance metrics.

\section{Multi-level MDO Algorithm}

The mathematical background of the algorithm driving the MDO analysis is discussed here. It mathematically coordinates the interactions among the various discipline level optimizations and the top system level optimization. A flow chart of the optimization process is presented in Figure 1. At the beginning of every iteration of the top level optimization, the discipline level optimizations are conducted first using as starting point the values for the design variables originating from the current step of the top level optimization. The vector $D V_{i}^{\text {starting }}$ represents the design variables associated with optimization discipline " $i$ ". Parameters $F_{1}, \ldots, F_{i-1}, \ldots, F_{i+1}, \ldots, F_{N}$ comprise functionals that have been computed in all other disciplines during the previous time that the discipline level computations were conducted and may be providing information needed in the computations of discipline " $i$ ". After the discipline level optimizations have been completed, the new values of the design variables $D V_{i}^{\text {optimal }}$ the values of the objective function at the starting point $O_{i}^{\text {start }}$ and at the optimal point $O_{i}^{\text {optimal }}$, and the value of a functional $F_{i}$ which is evaluated during the computations of discipline " $i$ " and used in the computations of another discipline are provided back to the top level optimization. Based on the results collected at the top level from the discipline level optimizations a targeted value is computed for each design variable of each discipline using Equation (1). Since any design variable may be shared among multiple disciplines, the target values for the design variables are determined based on the improvement encountered in the discipline level objective functions during the last optimization:

$$
d v_{\mathrm{j}}^{\text {target }}=\frac{\sum_{i=1}^{N}\left(d v_{i, j}^{\text {optimal }}\left|O_{i}^{\text {start }}-O_{i}^{\text {optimal }}\right|\right)}{\sum_{i=1}^{N}\left|O_{i}^{\text {start }}-O_{i}^{\text {optimal }}\right|}
$$

In Equation (1), the subscript " $j$ " refers to the $\mathrm{j}$-th design variable, $N$ is the total number of disciplines that share this design variable, and the superscripts "start" and "optimal" indicate starting and optimal values of design variables and objective functions for the " $i$ " discipline. In this manner if a design variable is shared by multiple disciplines, its targeted value is influenced the most by the discipline which encountered the largest improvement. The targeted values for the design variables are utilized when defining the objective function for the top level optimization. The top level objective function $O_{T}$ is augmented to include information about the target values of the design variables from all disciplines resulting in a minimization statement of:

$$
\begin{gathered}
\min \left(O_{T}+\sum_{j=1}^{J}\left|d v_{j}^{\text {target }}-d v_{j}\right|\right) \\
D V_{T}, d v_{j} j=1, \ldots, J
\end{gathered}
$$

where $J$ is the total number of design variables encountered in all disciplines and $D V_{T}$ are the design variables of the top, system level optimization. Any of the discipline level design variables can also be part of the vector of the system level design variables $D V_{T}$. In this manner, the influence of the discipline objective functions is considered by requiring the design variables at the top level to match the target values of the design variables which are involved in the discipline level optimizations while at the same time improving the system level objective function. 


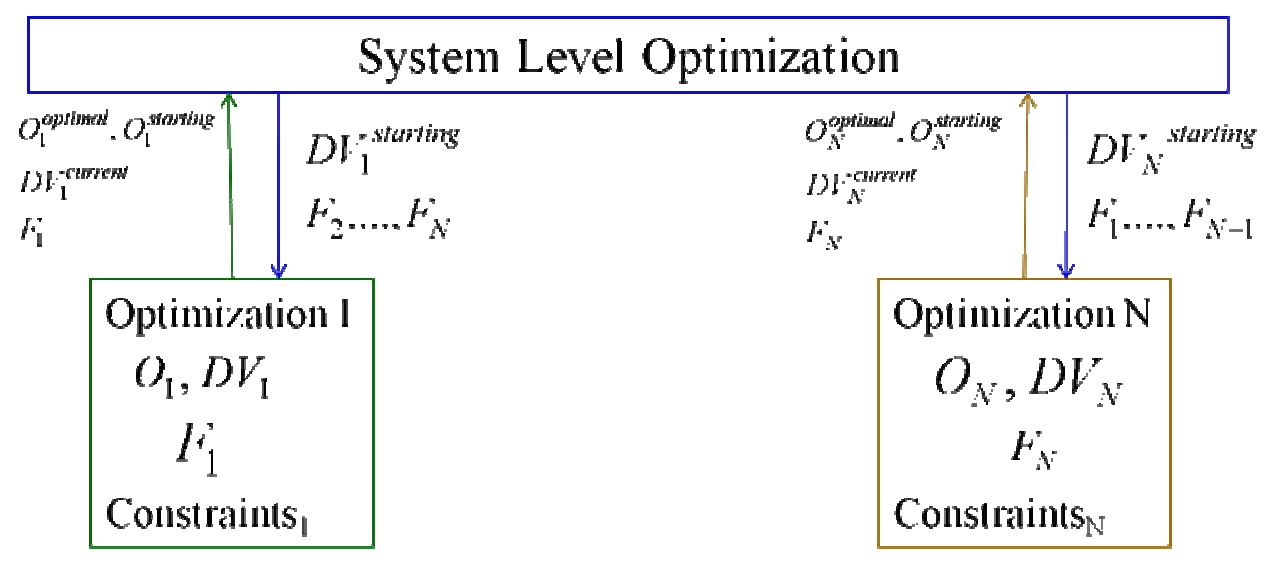

\section{Top Level Optimization:}

$$
d v_{\mathrm{i}}^{\text {1arget }}=\frac{\sum_{i=1}^{N}\left(\alpha v_{i, j}^{\text {optimal }} \mid O_{j}^{\text {start }}-O_{i}^{\text {optimat }}\right)}{\sum_{i=1}^{N}\left|O_{i}^{\text {start }}-O_{i}^{\text {optimat }}\right|}
$$

$$
\begin{gathered}
\min \left(O_{T}+\sum_{j-1}^{J}\left|d v_{i}^{\text {target }}-d v_{j}\right|\right) \\
D V_{T,} d v_{j} \quad j-1, \ldots, j \\
\text { Constraints } \\
+
\end{gathered}
$$$$
\text { Constraints } \mathbf{1}_{1}-1 . \quad . \mathrm{N}
$$

Figure 1. Flow chart of multi-level MDO algorithm

All the discipline constraints are also included in the top level optimization along with the top level constraints. In this manner the optimal point determined by the system level optimization will also be a feasible point for all disciplines.

$$
\begin{aligned}
g_{T}\left(D V_{T}\right) & \leq 0 \\
h_{T}\left(D V_{T}\right) & =0 \\
g_{i}\left(D V_{i}\right) & \leq 0 \\
h_{i}\left(D V_{i}\right) & =0
\end{aligned}
$$

where $g_{T}\left(D V_{T}\right)$ and $h_{T}\left(D V_{T}\right)$ are the inequality and equality constraints for the top level, respectively, and $g_{i}\left(D V_{i}\right)$ and $h_{i}\left(D V_{i}\right)$ are the inequality and equality constraints for the " $i$ " discipline. Interaction among multiple discipline level optimizations is coordinated through a top level optimization statement. Typically, the top level optimization addresses a global, overall system metric (such as cost, weight, etc.), while the discipline level optimizations target improvement in different performance attributes of a system. Each discipline has its own objective function, constraints, and design variables. Different disciplines can share common design variables and it is also possible for a "functional" evaluated within a particular discipline to influence computations in another discipline. The communication of the information among all the disciplines is coordinated through the top level optimization. This process allows coordination of the multiple discipline optimizations by the top level and facilitates the flow of information among disciplines.

\section{Gearbox foundation design}

In order to demonstrate the functionality provided by the network of optimizations capability in designing the structure of a vehicle under multiple disciplines, an optimization analysis for a rotorcraft gearbox foundation is presented. The weight of the gearbox base support structure of the rotorcraft fuselage presented in Figure 2 is minimized while at the same time the structural acoustics and the crash landing performances are optimizaed in parallel. The frame structure of the fuselage is presented in Figure 3, and the titanium gearbox base which is optimized is highlighted with light blue color. 


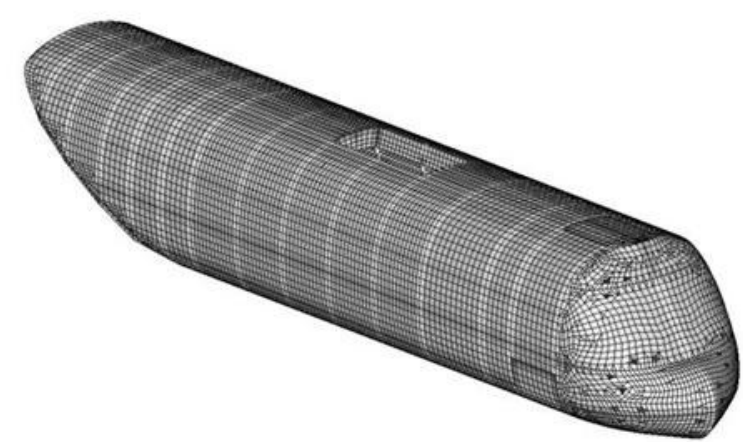

Figure 2. Finite Element Model of Rotorcraft Fuselage used in the Optimization Analysis

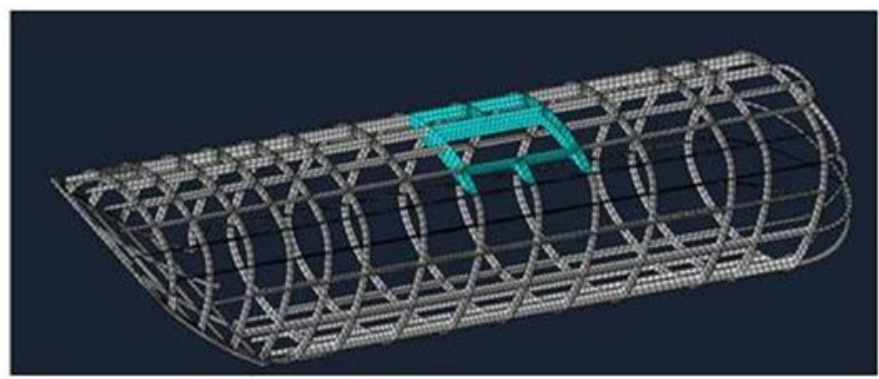

Figure 3. Frame of Rotorcraft Fuselage used in Optimization Analysis

Minimizing the weight of the titanium base where the gearbox is mounted comprises the system level objective. Two discipline optimizations are solved in parallel based on guidance and organization provided by the top level optimization. The two disciplines are associated with crashworthiness performance and with structural acoustics considerations, respectively. Figure 4 presents the flow chart of the MDO analysis. Six design variables are considered in the optimization, each associated with the thickness of a section of the titanium base. The six design variables are highlighted with different colors at the top level optimization in Figure 4. They are:

$\mathrm{t} 1$ : thickness of front panel (deep blue)

t2: thickness of rear panel (light blue)

t3: thickness of outside panel (green)

t4: thickness of cross stiffener panel (red)

t5: thickness of inner-front panel (yellow)

t6: thickness of inner-rear panel (orange)

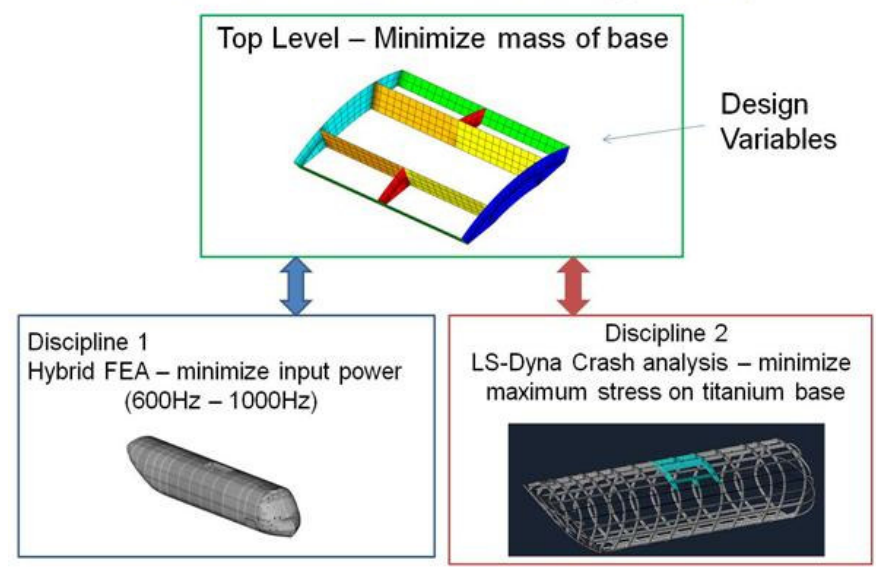

Figure 4. Flow chart of geabox base optimization analysis 
The discipline associated with the rotorcraft structural acoustics analysis is using as a simulation driver the hybrid FEA method [4-6]. The hybrid FEA has been developed for structure-borne structural acoustics simulations of systems comprised by stiff, loadbearing members and flexible panels. It combines the energy finite element method for modeling the flexible behavior of the panels with conventional finite elements for modeling the behavior of all remaining members. The utilization of the energy finite element method eliminates the requirement of using a very dense finite element model for capturing the flexible behavior of the panels at the relatively high frequency range of gearbox forcing excitation. In this application, the helicopter model is excited by unit harmonic forces in $\mathrm{x} / \mathrm{y} / \mathrm{z}$ directions applied at 4 locations where the gearbox is mounted on the titanium base (Figure 5). The forces at each node have 90 degree phase shift from the forces at its neighboring node. The objective is to minimize the overall input power to the helicopter structure under the prescribed excitation in the frequency range of $700 \mathrm{~Hz}$ to $1,000 \mathrm{~Hz}$. This type of structural dynamic objective is associated with fatigue considerations, structural vibration and interior noise concerns associated with passenger comfort.

The discipline associated with the crashworthiness is using LS-Dyna as the simulation driver. It simulates the helicopter being dropped to a rigid ground with impact velocity of $30 \mathrm{~m} / \mathrm{s}$. The objective is to minimize the maximum dynamic stress observed at the titanium base structure during the crash analysis. This objective ensures that the frame will not brake and the gearbox will not penetrate into the cabin during crash landing. A large concentrated mass is attached to the gearbox base representing the mass of the gearbox.

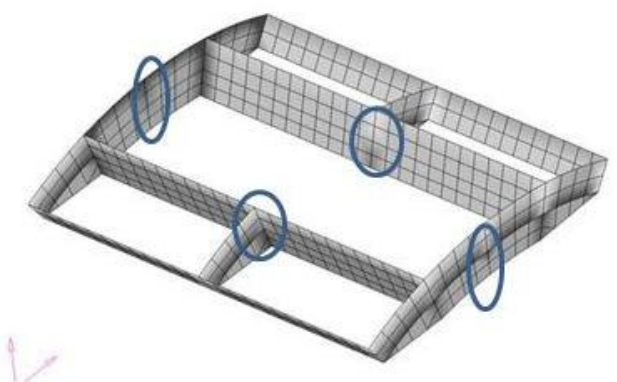

Figure 5. Locations on the titanium gearbox base where the gearbox excitation is applied

In all optimizations the six thickness parameters are ranging between $70 \%$ and $130 \%$ of their initial values. In the two discipline optimization a constraint is also imposed limiting the increase in the total mass of the base allowed by the discipline level optimizations. Within each iteration of the top level optimization, each discipline optimization is solved completely (i.e. minimizing the input power and minimizing the maximum dynamic stress) and the results are passed to the top level. The top level optimization consolidates the answers and at the same time drives the design in a direction that improves its own objective (i.e. minimizing the mass). The network of optimizations analysis provides the following results for the six design variables and for the objective functions of the top level and the two discipline level optimizations:

\begin{tabular}{|l|r|r|r|r|r|r|}
\hline & \multicolumn{7}{|c|}{ Normalized Thickness } \\
\hline & $\mathrm{t} 1$ & \multicolumn{1}{|c|}{$\mathrm{t} 2$} & $\mathrm{t} 3$ & $\mathrm{t} 4$ & \multicolumn{1}{c|}{$\mathrm{t} 5$} & \multicolumn{1}{c|}{$\mathrm{t} 6$} \\
\hline Initial & 1.0 & 1.0 & 1.0 & 1.0 & 1.0 & 1.0 \\
\hline MDO & 1.2997 & 1.2997 & 0.7003 & 1.295 & 0.7465 & 0.7003 \\
\hline
\end{tabular}

Table 1. Summary of the design variables from the network of optimizations analysis

\begin{tabular}{|c|c|c|c|}
\hline & $\begin{array}{c}\text { Mass reduction of gearbox } \\
\text { base }\end{array}$ & $\begin{array}{c}\text { Reduction of total input } \\
\text { power }\end{array}$ & $\begin{array}{c}\text { Reduction of maximum dynamic } \\
\text { stress }\end{array}$ \\
\hline $\begin{array}{c}\text { Optimal } \\
\text { Configuration }\end{array}$ & $9.02 \%$ & $15.6 \%$ & $25 \%$ \\
\hline
\end{tabular}

Table 2. Summary of improvement in the objective functions for the optimal configuration

For an easier interpretation of the results, the thicknesses that correspond to the optimal configuration are presented in Figure 6. The results are presented in a non-dimensional scale (1 indicates no change from initial values, values smaller than 1 indicate a decrease, and values larger than one indicate an increase). As it can be observed from 
Table 2 the mass of the titanium base of the gearbox is reduced by $\sim 9 \%$, while at the same time the vibrational input power is reduced by $\sim 15 \%$, and the maximum stress is reduced by $\sim 25 \%$ from the initial configuration.

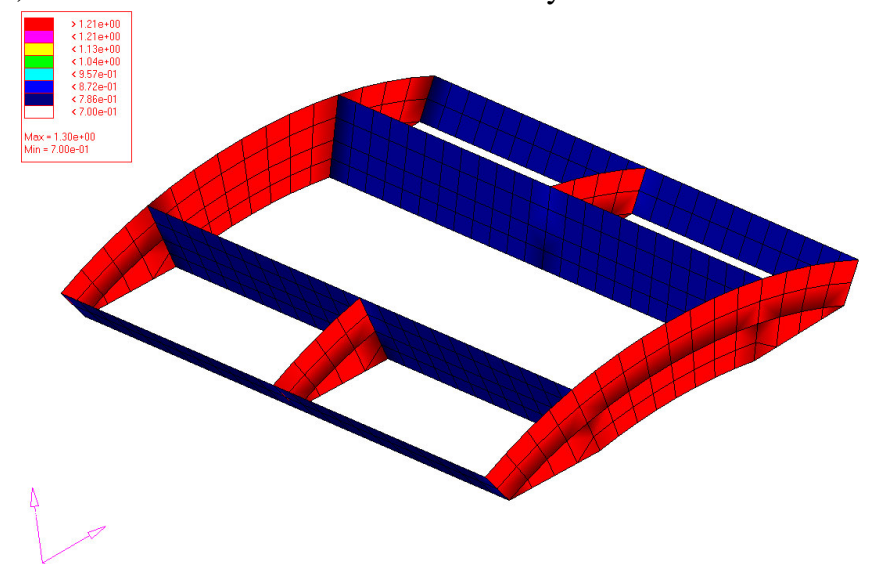

Figure 6. Optimal distribution of thicknesses for titanium gearbox base (non-dimensional)

The optimal design increases the thicknesses at the front and aft transverse panels and the cross stiffeners and reduces the thicknesses in all of the longitudinal panels towards the respective bounds. In order to investigate the physics that led to the optimal configuration the performance of the initial and the optimal designs are compared further. Figure 7 depicts the input power into the rotorcraft structure for the original and for the optimal configurations. It can be observed that the optimal design exhibits a significantly reduced input power within the requency range of $930 \mathrm{~Hz}-1,000 \mathrm{~Hz}$. To better understand the effect of the changes induced by the multi-discipline optimization analysis the vibration levels at the corresponding frequencies of maximum input power for the base are plotted in Figures 8 and 9 in the same scale for the original and the optimal configurations, respectively. It is clear that the optimal design has reduced vibration levels at the four excitation locations and therefore, reduced input power to the rotorcraft structure. Increasing the thickness of the forward and aft transverse members results in reducing the high vibration levels observed initially at the forward and aft mount locations. Incresing the thickness of the cross stiffeners reduces the vibration at the two transverse mount locations. The vibration of the longitudinal members can be increased without an impact to the input power as long as the vibration at the mount locations remains low. Thus, the thickness of the longitudinal members is reduced.

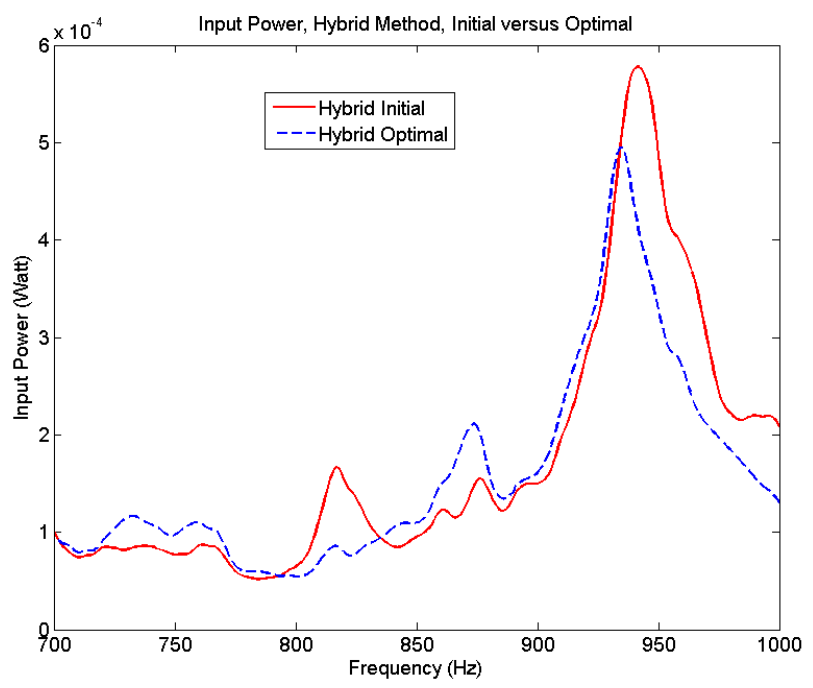

Figure 7. Input power for the $700 \mathrm{~Hz}-1,000 \mathrm{~Hz}$ range for the initial and the optimal designs 


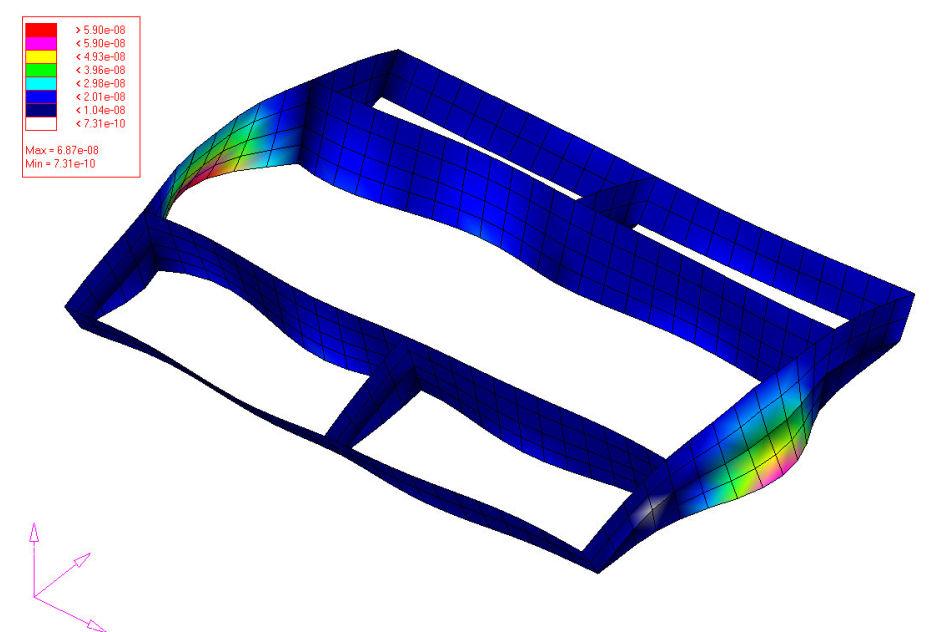

Figure 8. Vibration levels at base for initial design at frequency of maximum input power

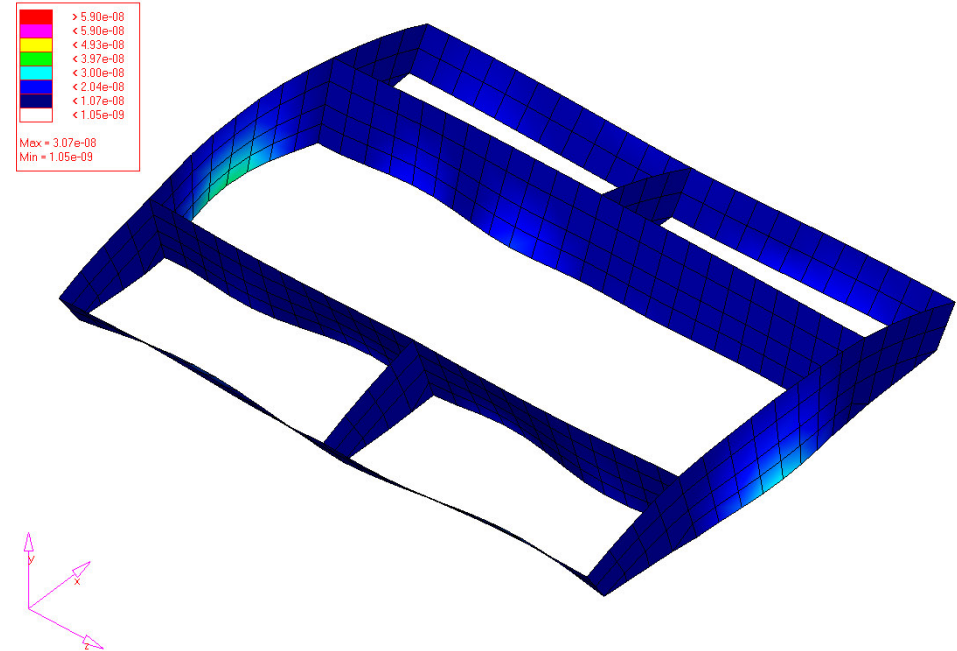

Figure 9. Vibration levels at base for the optimal design at frequency of maximum input power

For the crash analysis, the contour plots of the dynamic Von Mises stress are plotted in Figures 10 and 11 for the time step that exhibits the maximum dynamic stress in the initial and optimal configurations, respectively. The same color scale is used in Figures 10 and 11. The time steps that the maximum stress is encountered are not the same between the two design configurations. It can be observed that considerably lower dynamic stresses are observed in the optimal configuration.

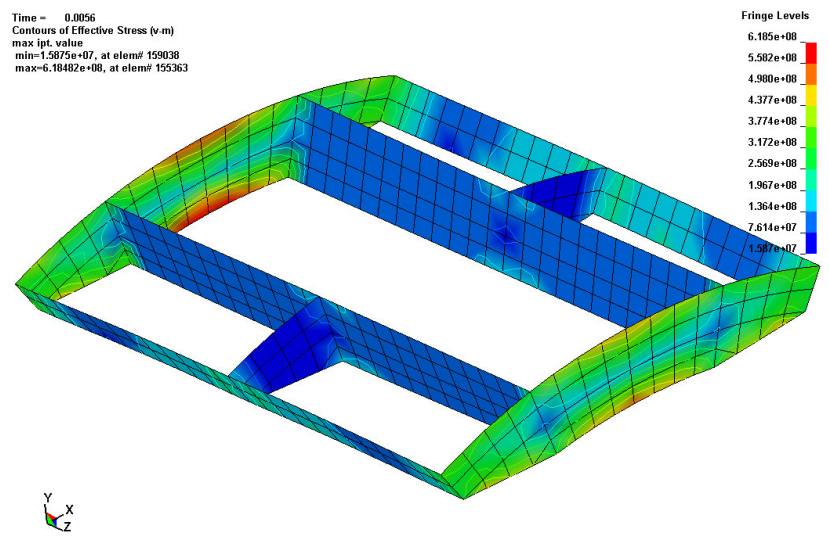

Figure 10. Stress distribution at the base during the time step when the maximum dynamic stress is encountered in the initial design 


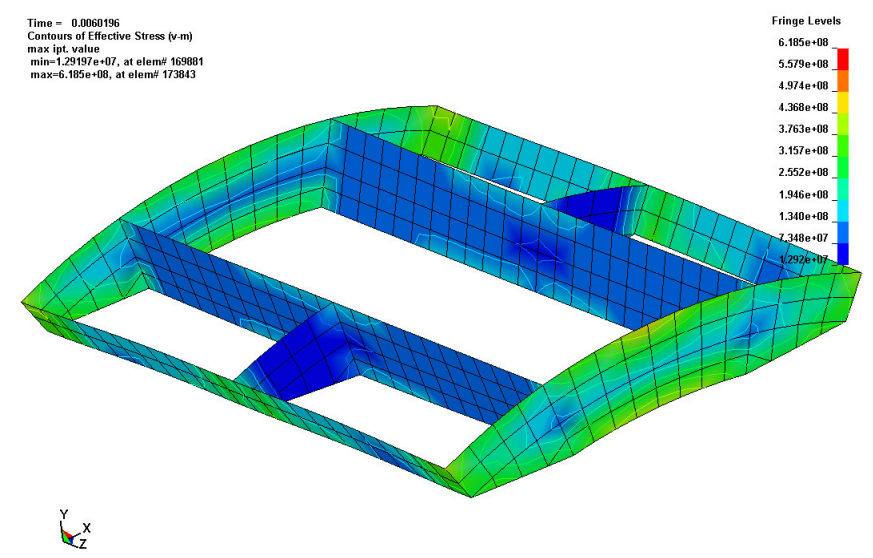

Figure 11. Stress distribution at the base during the time step when the maximum dynamic stress is encountered in the optimal design

Incresing the thicknesses of the transverse members leads to a reduction of the maximum dynamic stress, while the lower stresses exhibited in the longitudinal members allow to reduce their thicknesses. Overall it is demonstrated that the multi-discipline optimization analysis conducted using the multi-level MDO algorithm presented in Section I captures the interactions among the multiple and mutually competing disciplines, and guides the design to an optimal point that improves the system level objective while simultaneously improving the performance in all disciplines.

\section{Summary}

The application of a new multi-level MDO algorithm for a generic rotorcraft foundation design is presented. The system level requirements of reducing the mass of the foundation are conflicting with the discipline level requirements of reducing the input power into the system for structural-acoustics concerns, and reducing the maximum dynamic stress encountered under crash landing conditions. The new algorithm guides simultaneously the three optimization analyses and identifies a feasible design that improves all three objectives. A physical insight is gained by comparing the response of the intitial and the optimal configurations.

\section{Acknowledgments}

The rotorcraft analysis was conducted as part of the NASA Phase I SBIR contract NNX09CF14P (TPOC: Noah Schiller).

\section{References}

${ }^{1}$ Frank, P. D., A. J. Booker, et al. (1992). "A comparison of optimization and search methods for multidisciplinary design." AIAA Paper: 92-4827.

${ }^{2}$ Alexandrov, N. M. and M. Y. Hussaini (1997). Multidisciplinary Design Optimization: State of the Art, Society for Industrial \& Applied Mathematics.

${ }^{3}$ Yi, S. I., J. K. Shin, et al. (2008). "Comparison of MDO methods with mathematical examples." Structural and Multidisciplinary Optimization 35(5): 391-402

${ }^{4}$ S.B. Hong, A. Wang, N. Vlahopoulos, “A Hybrid Finite Element Formulation for a beam plate system," Journal of Sound and Vibration, Vol. 298, 2006, pp. 233 - 256.

${ }^{5}$ N. Vlahopoulos, S. Li, M.Viktorovitch, D. Caprioli, "Validation of a Hybrid Finite Element Formulation for Mid-Frequency Analysis of Vehicle Structures," SAE Paper 2007-01-2303, 2007 SAE Noise and Vibration Conference.

${ }^{6}$ R. Sbragio, A. Wang, N. Vlahopoulos, D. Caprioli, C. Bertolini, "Structure-borne Vehicle Analysis using a Hybrid Finite Element Method,” SAE Paper 2009-01-2196, 2009 SAE Noise and Vibration Conference. 